\title{
Functional Imaging-guided Radiotherapy for Locally Advanced Non- Small-Cell Lung Cancer: Review
}

\author{
Xiao Wang*, Yin Zhang, Ning Yue, Ke Nie
}

Department of Radiation Oncology, Rutgers-Cancer Institute of New Jersey, Robert Wood Johnson Medical School, New Brunswick, NJ, USA

Article Info

\section{Article Notes}

Received: August 10, 2018

Accepted: September 10, 2018

\section{*Correspondence:}

Dr. Xiao Wang, Ph.D., Department of Radiation Oncology, Rutgers-Cancer Institute of New Jersey, Robert Wood Johnson Medical School, New Brunswick, NJ 08904, USA; E-mail: xw240@cinj.rutgers.edu.

(c) 2018 Wang X. This article is distributed under the terms of the Creative Commons Attribution 4.0 International License.

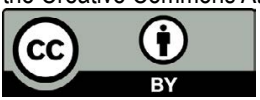

\section{Keywords:}

NSCLC

Functional Imaging

4DCT

High Functioning Lung

Photon Radiotherapy

Proton Radiotherapy

\section{ABSTRACT}

In treating locally advanced non-small-cell lung cancer (NSCLC), radiotolerance of the normal lung often limits the amount of dose that can be delivered to the primary cancer site. Radiation-related pneumonitis (RP) and other normal lung tissue complications have a significant impact on clinical outcome and patient quality of life. How to minimize treatment side effects while achieving desirable local control of lung cancers has been a continuous challenge.

Functional imaging-guided radiotherapy, which can achieve a local boost of primary cancer site or functional avoidance of normal organs, has been increasingly utilized in clinics. Various imaging approaches have been employed to achieve functional imaging guidance and implemented in different treatment regimens. There are several on-going clinical trials aiming to evaluate the clinical outcomes that are utilizing functional imaging-guided photon radiation to spare the high functioning portions of the lungs. The main applications of functional imaging-guided radiotherapy in the management of NSCLC patients will be discussed in this review.

\section{Introduction}

Radiotherapy plays a major role in the treatment of patients with locally advanced non-small-cell lung cancer (NSCLC), primarily due to the extent of the disease not suitable for surgery. Despite advances in local and systemic therapies, local control and survival remain poor and there is a sense that a therapeutic plateau has been reached with conventional approaches. On the contrary, strategies for dose escalation have shown encouraging results with improved therapeutic ratio and survival as confirmed by Radiation Therapy Oncology Group (RTOG) 9311 and several other clinical trials $^{1,2}$. However, the radio-tolerance of the normal lung is the primary limiting factor of the dose escalation to the primary cancer site. Therefore, one proposal to minimize the radiation-induced lung injury is to take consideration of the pulmonary function by deliberately reducing dose to highly functional regions when generating the treatment plan. This concept of functional imagingguided lung avoidance treatment has been investigated with several imaging modalities, including single photon emission computed tomography (SPECT), hyperpolarized (HP) gas magnetic resonance imaging (MRI), and increasingly with 4-dimensional (4D) computed tomography (CT) based measures of lung function. Here we review the applications with different functional imaging modalities and different treatment modalities. 


\section{Functional Lung Imaging Modalities}

Lung function can be evaluated by functional imaging modalities such as SPECT ${ }^{3,4}$, HP Helium or Xenon MRI ${ }^{5,6}$ and 4D CT ventilation imaging ${ }^{7-9}$.

Perfusion SPECT has long time been the most commonly used imaging modality for functional lung assessment ${ }^{3,4}$. The ventilation map generated by SPECT can be further utilized to optimize beam directions for radiation treatment planning to potentially spare highly functional normal lung $^{3,10}$. However, SPECT has limitations with low spatial and temporal resolution compared to anatomical imaging as CT or MRI. It also suffers from the potential errors in photon attenuation and scatters correction, imaging registration to CT for planning, and patient repositioning or setup inconsistency ${ }^{11}$. Recently HP gas MRI has been developed rapidly, including helium- $3^{5}$ with stronger signals due to higher levels of polarization and higher gyromagnetic ratio, and xenon- $129^{6}$, with an unlimited supply in nature and its falling cost, providing a unique tool for direct assessment of lung ventilation ${ }^{12}$. But in general, functional MRI is not yet widely available in clinics, and the routine application in clinical practice would be associated with long scanning time and high cost to patients ${ }^{13}$.

CT especially 4D-CT, on the contrary, is gaining its popularity to assess the pulmonary function due to the wide accessibility, routine clinical application, and low cost. Previous literature has detailed the methodology of deriving pulmonary ventilation maps from $4 \mathrm{D}-\mathrm{CT}^{7,9}$. Typically, a deformable image registration (DIR) is involved to obtain a displacement vector field (DVF) from peakexhale to peak-inhale phases from the 4D series. With the assumptions that regional ventilation is proportional to the regional volume change, either Hounsfield unit (HU) change $^{14-16}$ or Jacobian measurement ${ }^{7,17,18}$ of the DVF can be used to derive ventilation map. Currently, various DIR algorithms have been developed and tested, of which the transformation model ranges in complexity from a simple extension of a global affine transformation to a completely local or free-form model where each voxel in the image can move independently. Also, there are two classes of similarity metrics commonly used to regulate DIR performance: geometry-based or intensity-based. As 4D-CT pulmonary ventilation images can vary widely with DIR algorithms and metrics, careful validation is needed before the clinical use. Cui et al. have evaluated 7 different DIR algorithms in generating 4D-CT based lung ventilation maps and compared the results with what is captured by HP gas tagging MRI on three healthy patients ${ }^{19}$. A large number (300-500) of uniformly distributed landmarks were identified to enable a complete assessment of DIR throughout the entire lung. The 7 DIR method platforms included Velocity, MIM, Mirada, Elastix and 3 other inhouse built algorithms from DIRART toolbox such as
Double Force Demons, Improved Lucas-Kanade, and Iterative Optical Flow. Among all algorithms, the Jacobian derivation of the deformable vector fields (DVFs) generated from Velocity (multi-pass free-form deformation) gave the most reasonable result. Brennen et al. performed clinical validation by comparing using HU-based and Jacobianbased 4D-CT ventilation metrics with pulmonary function test data on ninety-eight lung cancer patients ${ }^{20}$. They confirmed HU-based ventilation metrics produced better correlations when compared to Jacobian-based ventilation metrics. There are also several studies attempting to validate 4DCT-ventilation by comparing it against other ventilation imaging modalities such as nuclear medicine ventilation-perfusion imaging ${ }^{21}$, xenon-CT ${ }^{4,17}, \mathrm{PET}^{22}$, and $\mathrm{MRI}^{23,24}$ or directly with pulmonary function testing ${ }^{20}$. The studies generally found good agreement on a global level, yet the regional physiologic accuracy has not been validated in patients. In addition, temporal changes in regional ventilation to a segment of lung previously impaired by compression from a local tumor might occur during the course of radiation treatment. A possible explanation of these changes is that the shrinkage of lung tumor in response to radiation might increase the ventilation due to the reopening of the airways ${ }^{25}$. Nevertheless, additional work is needed to validate the regional physiologic accuracy of 4DCT derived ventilation imaging in real patients especially during the course of radiation treatment.

\section{Implementations with Photon Radiotherapy}

The feasibility of photon treatment planning using an optimal beam arrangement aiming at preserving the high functioning portion of the lung under the guidance of functional imaging has been widely studied. Studies have demonstrated decreasing the radiation dose to highfunctioning lung areas and directing the radiation to the parts with inactive perfusion/ventilation may help to protect highly functioning lung regions and thus reduce the incidence and seriousness of radiation pneumonitis $(\mathrm{RP})^{7,8,26-30}$. Those treatment plans did not compromise the DVHs of OARs, such as the spinal cord, esophagus, and heart, which may be additional important clinical factors. These results also demonstrated that functional imaging could be applied safely to photon radiation treatments for patients with NSCLC, without exceeding the dose-volume tolerances of OARs.

In addition, Ireland et al. showed that patients with specific types of functional defects, tumor volumes and positions will benefit from the inclusion of functional data for normal lung dose reduction ${ }^{31}$. Lavrenkov et al. compared IMRT planning and 3D-CRT planning using functional SPECT perfusion images and demonstrated that IMRT planning led to a lower high functioning lung mean dose than 3D-CRT planning ${ }^{32}$. Yamamoto et al. demonstrated that $4 \mathrm{D}-\mathrm{CT}$ ventilation imaging based functional IMRT and 
VMAT treatment planning, by changing only one variable (i.e., absence/presence of constraints on the functioning lung), led to significant reductions in the high functioning lung dose ${ }^{27}$. Faught et al. discussed which dose-function metrics should be used for treatment planning and aimed to construct most predictive models to predict $\mathrm{RP}^{33}$.

There are several on-going clinical trials, such as NCI NCT02308709, NCT02528942, and NCT02843568, that utilize functional imaging-guided photon radiation to spare higher functioning portions of the lung and aim to evaluate the clinical outcome. With this technique of functional lung sparing, the goal is to reduce the rates of lung parenchymal toxicity, i.e. pneumonitis, and to further allow for dose escalation.

\section{Implementations with Proton Radiotherapy}

To date, most of the work utilizing functional imaging was done with photon-based radiotherapy and limited work was reported with proton treatment. The energy deposition of protons has theoretical advantages because of the physical property of proton particles, which can be exploited to reduce exposure of normal tissues to radiation, particularly to reduce low dose radiation exposure to OARs. Under this premise, emerging dosimetric and clinical studies are being undertaken to assess the role of proton radiotherapy vs. photon and the potential to further escalate dose with proton treatment ${ }^{34-38}$.

Our group, is the first to demonstrate the feasibility of incorporating 4DCT-based ventilation map into proton planning with both double scattering (DSPT) and pencil scanning (IMPT) techniques ${ }^{39}$. The results showed that both DSPT and IMPT plans gave a superior dosimetric advantage over photon IMRTs in sparing low dose regions of the total lungs in terms of V5 (volume receiving $\geq 5 \mathrm{~Gy}$ ). The functional planning in IMPT delivery can further reduce the low dose in high functioning lung without degrading the PTV dosimetric coverage or increasing dose to critical structures. Yet, the functional DSPT only showed marginal benefit in sparing high-functioning lung in terms of V5 or V20 (volume receiving $\geq 20 \mathrm{~Gy}$ ) compared to anatomical plans. Recently, O'Reilly et al. conducted a case-control study on 48 NSCLC patients to compare the high-ventilation lung dose with RP outcome ${ }^{40}$. Their data supported findings that dose to the high-ventilated lung may serve as a predictor of RP regardless treated with photons or protons.

To our best knowledge, functional imaging-guided proton treatment with normal lung avoidance is still very new and no clinical trial has been conducted so far. With the rapid growth of proton centers and advances in imaging techniques, there will be more interests in applying functional imaging in proton planning. However, for lung proton treatment especially pencil beam delivering, several practical issues remain. For the IMPT technique, one of the major concerns comes from the interplay effects between the moving beams and moving tissue. The magnitude of the interplay effect with scanning proton beams has been reported in previous studies, and it has been shown that proton dose could be impacted enormously by the interplay effect for tumor motions around or larger than $10 \mathrm{~mm}^{41-}$ 45. Kardar et al. introduced a 4D dynamic dose simulator and further investigated the impact of motion pattern and starting phases on the interplay effects ${ }^{46,47}$. They observed situations in which motion more than $5 \mathrm{~mm}$ and small tumor sizes led to relatively large uncertainties caused by the interplay effect in a single fraction. In contrast, for some patients with motion less than $5 \mathrm{~mm}$ and large tumor size, the interplay effect was small. Moreover, a recent study by Inoue et al. evaluated the impact of setup and range uncertainties, breathing motion, and interplay effects in IMPT dose distributions ${ }^{48}$. Their results demonstrated that in robust-optimized plans the dosimetric effects due to geometric and radiologic variation had a limited impact on target coverage, target dose homogeneity, and OAR dose parameters when treated with multi-fractionation clinical scheme. As such for future functional imaging-guided IMPT delivery, it is very critical to identify a proper patient cohort. It might be appropriate to include only patients with nonsmall size tumor and also with breathing motion less than 5-7 mm. In addition, as suggested by Kardar et al. and Li et $a l^{46,47}$, a target coverage difference between the maximum in-hale (CT0) and maximum ex-hale (CT50) needs to be assessed and a less than $5 \%$ difference may suggest a robust coverage. Nevertheless, further studies are needed to ensure the robustness of the proton treatment for moving target.

\section{Conclusion}

This review highlights the inclusion of normal lung functional data into treatment planning using photon or proton radiation for lung cancer. It is feasible to use functional imaging techniques to obtain the perfusion/ ventilation imaging to assess the normal lung function and then optimize the treatment planning by limiting dose to the functional lung regions. The benefit is to minimize the risk of radiation-induced lung injury, which may potentially allow dose escalation and thereby improve overall survival. Several clinical trials with photon treatment are on-going and similar studies are expected to extend to proton treatment. However, beyond the question regarding which imaging or treatment modality should be used for functional imaging-guided radiation treatment, the clinical implication underlying the modification of the dose for functional lung should be investigated. The clinical assumption for dose-redistribution to avoid high-functional lung is that a higher dose can be targeted to low perfused/ ventilated lung. Lung function can be reduced irreversibly by radiation, but tumor itself can be responsible for reduced 
lung function. Therefore, a potential limitation of normal lung avoidance is that lung volumes that may have received a functionally modified, amplified dose may regain some degree of function after treatment. Hence, whether defects are permanent or reversible becomes an important issue when assigning functional and non-functional planning constraints. Further validation tests, planning studies and clinical trials will be required to increase our understanding of the potential benefits and long-term effects of functional imaging-guided lung avoidance planning strategies.

\section{Acknowledgements}

This work is supported by the Rutgers-RBHS precision medicine pilot grant, the Rutgers Cancer Institute of New Jersey (Grant No. P30CA072720).

\section{References}

1. Bradley J, Graham MV, Winter K, et al. Toxicity and outcome results of RTOG 9311: A phase I-II dose-escalation study using threedimensional conformal radiotherapy in patients with inoperable non-small-cell lung carcinoma. International Journal of Radiation Oncology* Biology* Physics. 2005; 61(2): 318-328.

2. Socinski MA, Morris DE, Halle JS, et al. Induction and concurrent chemotherapy with high-dose thoracic conformal radiation therapy in unresectable stage IIIA and IIIB non-small-cell lung cancer: A doseescalation phase I trial. Journal of clinical oncology. 2004; 22(21): 4341-4350.

3. Shioyama Y, Jang SY, Liu HH, et al. Preserving functional lung using perfusion imaging and intensity-modulated radiation therapy for advanced-stage non-small cell lung cancer [published online ahead of print 2007/04/21]. Int J Radiat Oncol Biol Phys. 2007; 68(5): 13491358.

4. Suga K. Technical and analytical advances in pulmonary ventilation SPECT with xenon-133 gas and Tc-99m-Technegas. Annals of nuclear medicine. 2002; 16(5): 303-310.

5. van Beek EJ, Wild JM, Kauczor HU, et al. Functional MRI of the lung using hyperpolarized 3-helium gas. Journal of Magnetic Resonance Imaging: An Official Journal of the International Society for Magnetic Resonance in Medicine. 2004; 20(4): 540-554.

6. Fain S, Schiebler ML, McCormack DG, et al. Imaging of lung function using hyperpolarized helium-3 magnetic resonance imaging: review of current and emerging translational methods and applications. Journal of Magnetic Resonance Imaging. 2010; 32(6): 1398-1408.

7. Yamamoto T, Kabus S, Klinder T, et al. Four-dimensional computed tomography pulmonary ventilation images vary with deformable image registration algorithms and metrics [published online ahead of print 2011/04/28]. Med Phys. 2011; 38(3): 1348-1358.

8. Faught AM, Miyasaka Y, Kadoya N, et al. Evaluating the toxicity reduction with computed tomographic ventilation functional avoidance radiation therapy. International Journal of Radiation Oncology* Biology* Physics. 2017; 99(2): 325-333.

9. Castillo R, Castillo E, Martinez J, et al. Ventilation from four-dimensional computed tomography: density versus Jacobian methods. Physics in Medicine \& Biology. 2010; 55(16): 4661.

10. Munawar I, Yaremko BP, Craig J, et al. Intensity modulated radiotherapy of non-small-cell lung cancer incorporating SPECT ventilation imaging. Medical physics. 2010; 37(4): 1863-1872.

11. Petersson J, Sánchez-Crespo A, Larsson SA, et al. Physiological imaging of the lung: single-photon-emission computed tomography (SPECT). Journal of applied physiology. 2007; 102(1): 468-476.
12. Patz S, Hersman FW, Muradian I, et al. Hyperpolarized 129Xe MRI: a viable functional lung imaging modality? European journal of radiology. 2007 ; 64(3): 335-344.

13. Ireland $\mathrm{RH}$, Bragg CM, McJury M, et al. Feasibility of image registration and intensity-modulated radiotherapy planning with hyperpolarized helium-3 magnetic resonance imaging for non-small-cell lung cancer. International Journal of Radiation Oncology* Biology* Physics. 2007; 68(1): 273-281.

14. Guerrero T, Sanders K, Castillo E, et al. Dynamic ventilation imaging from four-dimensional computed tomography. Physics in Medicine \& Biology. 2006; 51(4): 777.

15. Simon BA. Non-invasive imaging of regional lung function using $x$-ray computed tomography. Journal of clinical monitoring and computing. $2000 ; 16(5-6): 433-442$.

16. Vinogradskiy YY, Castillo R, Castillo E, et al. Use of weekly 4DCT-based ventilation maps to quantify changes in lung function for patients undergoing radiation therapy. Medical physics. 2012; 39(1): 289-298.

17. Reinhardt JM, Ding K, Cao K, et al. Registration-based estimates of local lung tissue expansion compared to xenon CT measures of specific ventilation. Medical image analysis. 2008; 12(6): 752-763.

18. Bayouth J, Du K, Christensen G, et al. Establishing a relationship between radiosensitivity of lung tissue and ventilation. International Journal of Radiation Oncology• Biology• Physics. 2012; 84(3): S31-S32.

19. Cui T. Evaluation of Lung Ventilation Maps with Hyperpolarized Gas Tagged Magnetic Resonance Imaging and its Application towards Respiratory Motion Modeling 2015.

20. Brennan D, Schubert L, Diot Q et al. Clinical validation of 4-dimensional computed tomography ventilation with pulmonary function test data [published online ahead of print 2015/03/31]. Int J Radiat Oncol Biol Phys. 2015; 92(2): 423-429.

21. Vinogradskiy Y, Koo PJ, Castillo R, et al. Comparison of 4-dimensional computed tomography ventilation with nuclear medicine ventilationperfusion imaging: a clinical validation study. International Journal of Radiation Oncology* Biology* Physics. 2014; 89(1): 199-205.

22. Kipritidis J, Siva S, Hofman MS, et al. Validating and improving CT ventilation imaging by correlating with ventilation 4D-PET/CT using 68Ga-labeled nanoparticles. Medical physics. 2014; 41(1).

23. Mathew L, Wheatley A, Castillo R, et al. Hyperpolarized 3He magnetic resonance imaging: comparison with four-dimensional x-ray computed tomography imaging in lung cancer. Academic radiology. 2012; 19(12): 1546-1553.

24. Tahir BA, Swift AJ, Marshall H, et al. A method for quantitative analysis of regional lung ventilation using deformable image registration of CT and hybrid hyperpolarized gas/1H MRI. Physics in Medicine \& Biology. 2014; 59(23): 7267.

25. Seppenwoolde Y, Muller SH, Theuws JC, et al. Radiation dose-effect relations and local recovery in perfusion for patients with nonsmall-cell lung cancer. International Journal of Radiation Oncology* Biology* Physics. 2000; 47(3): 681-690.

26. Yamamoto T, Kabus S, Bal M, et al. The first patient treatment of computed tomography ventilation functional image-guided radiotherapy for lung cancer. Radiotherapy and Oncology. 2016; 118(2): 227-231.

27. Yamamoto T, Kabus S, von Berg J, et al. Impact of four-dimensional computed tomography pulmonary ventilation imaging-based functional avoidance for lung cancer radiotherapy [published online ahead of print 2010/07/22]. Int J Radiat Oncol Biol Phys. 2011; 79(1): 279-288.

28. Vinogradskiy Y, Castillo R, Castillo E, et al. Use of 4-dimensional computed tomography-based ventilation imaging to correlate lung 
dose and function with clinical outcomes [published online ahead of print 2013/03/12]. Int J Radiat Oncol Biol Phys. 2013; 86(2): 366-371.

29. Wang R, Zhang S, Yu H, et al. Optimal beam arrangement for pulmonary ventilation image-guided intensity-modulated radiotherapy for lung cancer. Radiation Oncology. 2014; 9(1): 184.

30. Yin Y, Chen Jh, Li BS, et al. Protection of lung function by introducing single photon emission computed tomography lung perfusion image into radiotherapy plan of lung cancer. Chinese medical journal. 2009; 122(5): 509-513.

31. Ireland R, Tahir B, Wild J, et al. Functional image-guided radiotherapy planning for normal lung avoidance. Clinical Oncology. 2016; 28(11): 695-707.

32. Lavrenkov K, Christian JA, Partridge M, et al. A potential to reduce pulmonary toxicity: the use of perfusion SPECT with IMRT for functional lung avoidance in radiotherapy of non-small cell lung cancer. Radiotherapy and oncology. 2007; 83(2): 156-162.

33. Faught AM, Yamamoto T, Castillo R, et al. Evaluating which dosefunction metrics are most critical for functional-guided radiation therapy. International Journal of Radiation Oncology* Biology* Physics. 2017; 99(1): 202-209.

34. Seco J, Panahandeh HR, Westover K, et al. Treatment of non-small cell lung cancer patients with proton beam-based stereotactic body radiotherapy: dosimetric comparison with photon plans highlights importance of range uncertainty. International Journal of Radiation Oncology* Biology* Physics. 2012; 83(1): 354-361.

35. Welsh J, Gomez D, Palmer MB, et al. Intensity-modulated proton therapy further reduces normal tissue exposure during definitive therapy for locally advanced distal esophageal tumors: a dosimetric study. International Journal of Radiation Oncology* Biology* Physics. 2011; 81(5): 1336-1342.

36. Shioyama Y, Tokuuye K, Okumura T, et al. Clinical evaluation of proton radiotherapy for non-small-cell lung cancer. International Journal of Radiation Oncology* Biology* Physics. 2003; 56(1): 7-13.

37. Chang JY, Zhang X, Wang X, et al. Significant reduction of normal tissue dose by proton radiotherapy compared with three-dimensional conformal or intensity-modulated radiation therapy in Stage I or Stage III non-small-cell lung cancer [published online ahead of print 2006/05/10]. Int J Radiat Oncol Biol Phys. 2006; 65(4): 1087-1096.

38. Giaddui T, Chen W, Yu J, et al. Establishing the feasibility of the dosimetric compliance criteria of RTOG 1308: phase III randomized trial comparing overall survival after photon versus proton radiochemotherapy for inoperable stage II-IIIB NSCLC [published online ahead of print 2016/05/05]. Radiat Oncol. 2016; 11: 66.

39. Huang Q, Jabbour SK, Xiao Z, et al. Dosimetric feasibility of 4DCTventilation imaging guided proton therapy for locally advanced nonsmall-cell lung cancer. Radiation Oncology. 2018; 13(1): 78.

40. O'Reilly S, Jain V, Huang Q, et al. BEST IN PHYSICS (JOINT IMAGINGTHERAPY): Avoiding Highly Functional Ventilation Zones Decreases the Risk of Radiation Pneumonitis During Proton and Photon Radiotherapy for Lung Cancer. Paper presented at: AAPM 60th Annual Meeting2018; Nashville, TN.

41. Phillips MH, Pedroni E, Blattmann H, et al. Effects of respiratory motion on dose uniformity with a charged particle scanning method. Physics in Medicine \& Biology. 1992; 37(1): 223.

42. Lambert J, Suchowerska N, McKenzie D, et al. Intrafractional motion during proton beam scanning. Physics in Medicine \& Biology. 2005; 50(20): 4853.

43. Kraus K, Heath E, Oelfke U. Dosimetric consequences of tumour motion due to respiration for a scanned proton beam. Physics in Medicine \& Biology. 2011; 56(20): 6563.

44. Bert C, Grözinger SO, Rietzel E. Quantification of interplay effects of scanned particle beams and moving targets. Physics in Medicine \& Biology. 2008; 53(9): 2253

45. Grassberger C, Dowdell S, Lomax A, et al. Motion interplay as a function of patient parameters and spot size in spot scanning proton therapy for lung cancer. International Journal of Radiation Oncology* Biology* Physics. 2013; 86(2): 380-386.

46. Kardar L, Li Y, Li X, et al. Evaluation and mitigation of the interplay effects of intensity modulated proton therapy for lung cancer in a clinical setting. Practical radiation oncology. 2014; 4(6): 259-268.

47. Li Y, Kardar L, Li X, et al. On the interplay effects with proton scanning beams in stage III lung cancer. Medical physics. 2014; 41(2): 021721.

48. Inoue T, Widder J, van Dijk LV, et al. Limited Impact of Setup and Range Uncertainties, Breathing Motion, and Interplay Effects in Robustly Optimized Intensity Modulated Proton Therapy for Stage III Nonsmall Cell Lung Cancer [published online ahead of print 2016/09/30]. Int J Radiat Oncol Biol Phys. 2016; 96(3): 661-669. 